Revista Destaques Acadêmicos, Lajeado, v. 9, n. 4, 2017. ISSN 2176-3070 DOI: http://dx.doi.org/10.22410/issn.2176-3070.v9i4a2017.1657 www.univates.br/revistas

\title{
OBJETOS DE APRENDIZAGEM COMO FERRAMENTA DE APOIO PARA O ENSINO DE FÍSICA
}

\author{
Tales Igor Ebert ${ }^{1}$, Bruno Locatelli ${ }^{2}$, Eduardo Caron ${ }^{3}$, Heryk Gasparini ${ }^{4}$, \\ Andréia Spessatto De Maman ${ }^{5}$, Sônia Elisa Marchi Gonzatti ${ }^{6}$, Marcelo de \\ Gomensoro Malheiros ${ }^{7}$
}

Resumo: Este trabalho relata uma experiência pedagógica desenvolvida no âmbito do ensino superior, em uma disciplina de Física que integra o currículo dos cursos de Engenharia de Software e de Computação. Os estudantes foram desafiados a desenvolverem simulações computacionais, utilizando linguagens de programação com as quais já tivessem alguma familiaridade e que envolvessem alguns fenômenos físicos abordados na disciplina. A atividade foi concebida e orientada por duas professoras de física e por um professor da área de engenharia da computação. Neste artigo, são apresentados dois objetos de aprendizagem desenvolvidos por um grupo de estudantes dos cursos de Engenharia de Software e Engenharia da Computação da UNIVATES. Um deles explora o lançamento oblíquo e o outro, as condições de flutuação de corpos em certos fluidos. As principais linguagens de programação utilizadas foram PHP: (Hypertext Preprocessor), JS (JavaScript), C/C++ e plataforma Scratch. Quanto aos principais conceitos físicos explorados, foi necessário revisar as componentes horizontal e vertical de um movimento bidimensional, relacionando-os ao princípio da independência dos movimentos; como determinar o tempo total de voo do projétil e o seu alcance. Para o segundo objeto, o conceito-chave explorado com a simulação é a relação entre densidade média de um corpo e a do fluido no qual está submerso.

1 Graduando do Curso de Engenharia de Software da UNIVATES.

2 Graduando do Curso de Engenharia da Computação da UNIVATES.

3 Graduando do Curso de Engenharia de Software da UNIVATES.

4 Graduando do Curso de Engenharia da Computação da UNIVTES.

5 Licenciada em Ciências Exatas. Mestre em Ensino de Ciências Exatas, UNIVATES. Professora do CETEC.

6 Graduada em Ciências. Doutora em Educação, UNIVATES. Professora do CETEC.

7 Graduado em Engenharia de Computação Doutor em Computação, UNIVATES. Professor do CETEC 
Palavras-chave: Objeto de Aprendizagem. Física. Linguagem de programação. Simulação.

\section{INTRODUÇÃO}

Diariamente professores se deparam com o desafio que é ensinar estudantes com diferentes pensamentos e culturas na busca pelo conhecimento. Formar profissionais com habilidades de interpretar e modelar problemas e articular saberes de diferentes áreas está entre os fatores que os desafiam a repensar o ensino de Física.

Segundo Veit e Teodoro (2002), um ensino tradicional baseia-se na exposição de conceitos que transmitem uma ideia pouco motivadora do que é a ciência, logo esta metodologia já não é mais eficiente, seja nas escolas ou universidades. Além disso, a forma como o ensino de ciências é desenvolvido não representa a ciência como ela é concedida (MOREIRA, 2014). "As teorias e modelos científicos são ensinados como verdades, como "descobertas geniais", como definitivos, acabados. " (MOREIRA, 2014, p. 2). O autor enfatiza que os professores não apresentam os conhecimentos como construções científicas, e propõe o paradoxo de que "ensina-se ciências sem uma concepção do que é ciência" (Ibidem, 2014). Para Veit e Teodoro (2002), o Ensino de Física é afetado por esse tipo de abordagem,

Na prática a Física representa para o estudante, na maior parte das vezes, uma disciplina muito difícil, em que é preciso decorar fórmulas cuja origem e finalidades são desconhecidas. Um ensino de Física baseado na exposição de conceitos transmite uma ideia incompleta e pouco motivadora do que é a ciência e de como ela se constrói.

Outro fator presente na sala de aula são as tecnologias. Nosso jovem está o tempo todo conectado virtualmente e o computador e as novas tecnologias podem ser uma poderosa ferramenta didática. Muitas atividades poderiam ser desenvolvidas por meio de sua utilização, seja quando o aluno não pode vivenciar a situação de estudo, ou para desenvolver sua própria pesquisa, a visualização na tela do computador se torna uma maneira de construir o conhecimento. Brandão, Araújo e Veit (2008, p. 12) afirmam:

O computador, visto como uma ferramenta didática no auxílio da aprendizagem pode fornecer oportunidades ímpares para a contextualização, visualização e apresentações das mais diversas situações físicas que possam dar sentido ao conceito físico que esteja sendo trabalhado pelo professor.

Nesta perspectiva, este trabalho tem como objetivo apresentar dois objetos de aprendizagem que foram desenvolvidos por estudantes de 
Engenharia da Computação e Engenharia de Software durante um semestre letivo na disciplina de Física Mecânica e Fenômenos de Transporte, auxiliados por três professores, dois de Física e um de Computação, de uma universidade da região Sul do país.

Os objetos de aprendizagem foram criados com o propósito de auxiliar os alunos da disciplina na compreensão de conteúdos da Física por meio do uso de programação básica. Todos os objetos de aprendizagem foram desenvolvidos exclusivamente pelos estudantes em pequenos grupos durante o período de um semestre letivo. Foram criados nove objetos de aprendizagem durante a disciplina, neste trabalho serão apresentados dois deles. O primeiro refere-se ao cálculo de um lançamento oblíquo por meio de uma calculadora on-line e o outro explora a densidade em fluidos e as condições de flutuação de corpos nesses fluidos.

\section{DESENVOLVIMENTO}

Neste trabalho definem-se objeto de aprendizagem (AO) segundo a concepção de David A. Wiley, que considera como "qualquer recurso digital que pode ser reutilizado para apoiar a aprendizagem" (WILEY, 2000, p. 4, tradução nossa).

Segundo Rockenbach (2006), construir objetos de aprendizagem requer um complexo arranjo e habilidades multidisciplinares. Para conceber um objeto de aprendizagem é preciso: (a) conhecer a temática do problema; (b) determinar a abordagem pedagógica que norteará sua concepção e uso; (c) saber utilizar ferramentas para sua construção e (d) trabalhar de forma coerente para atingir os objetivos do projeto educacional. Logo, os objetos que serão apresentados neste trabalho exigiram dos alunos várias habilidades, desde o conhecimento de programação até o conhecimento dos fenômenos da física e as equações matemáticas necessárias para os cálculos e simulações.

Nesse trabalho, o primeiro objeto desenvolvido foi uma simulação para lançamentos de projéteis que descreve a trajetória parabólica de um projétil. Conforme Silva e Cotta (2015), uma partícula é lançada com velocidade inicial $\left(\mathrm{v}_{0}\right)$, segundo um ângulo (a) em relação ao eixo horizontal (lançamento oblíquo), estando sob a ação da aceleração da gravidade $(\mathrm{g})$, agindo verticalmente para baixo, impondo uma trajetória parabólica, resultante da composição de dois movimentos. O movimento de um projétil pode ser estudado tendo em consideração o Princípio da Independência dos movimentos, proposto por Galileu, em que os movimentos horizontais (retilíneo e uniforme) e verticais (retilíneo e uniformemente acelerado) podem ser analisados de modo independente. O movimento geral do projétil pode ser obtido pela sobreposição destes dois movimentos. O movimento parabólico é caracterizado por dois movimentos simultâneos em direções perpendiculares. 
Para a criação deste OA foi utilizado de um certificado SSL (Secure Socket Layer). Isso garante que os dados serão criptografados e somente o cliente e o servidor possuem a chave para acessar a informação (CERT.BR, 2015).

O simulador necessita que o usuário insira os dados como: ângulo de lançamento, velocidade inicial e gravidade local. Após a inserção destes dados, o simulador calcula outras informações como altura máxima atingida, tempo total do percurso no ar, tempo de subida até o ponto mais alto, entre outros, e ainda esboçam gráfico que representa o percurso do objeto em movimento. Os atritos são considerados sempre desprezíveis em todas as situações propostas.

O segundo Objeto de Aprendizagem simula as situações de blocos com determinadas massas, jogados em recipientes com determinados líquidos. As leis que regem a hidrostática estão presentes no nosso dia-a-dia, mais do que podemos imaginar. Elas se verificam, por exemplo, na água que sai da torneira das nossas residências, nas represas das hidrelétricas que geram a energia elétrica que utilizamos e na pressão que o ar está exercendo sobre você nesse exato momento. Para a construção desta ferramenta foi necessário um conhecimento prévio de hidrostática, que é a parte da física que estuda os líquidos e os gases em repouso, sob ação de um campo gravitacional constante, como ocorre quando estamos na superfície da Terra. O OA irá simular blocos que são jogados em um recipiente com água e verificar o comportamento deles dentro deste recipiente, ou seja, se ficam emersos, submersos ou parcialmente emersos.

\subsection{Estrutura do objeto de aprendizagem: lançamento oblíquo}

A ideia inicial foi de construir um simulador que fosse disponibilizado na internet, e para o bom potencial do mesmo, projetou-se uma pequena arquitetura capaz de permitir que o projeto seja futuramente expandido para outras áreas e simulações.

A Figura 1 ilustra como foi projetada a estrutura composta de três camadas, front-end, responsável em calcular as mais diversas variáveis em linguagens de programação distintas, o front-end, que pode ser implementado em diferentes linguagens conforme a necessidade, e por fim, a camada de webservice, a mais importante, que é responsável por fazer a comunicação entre as camadas de front e back-end. 
Figura 1: Arquitetura do objeto de aprendizagem lançamento de projétil

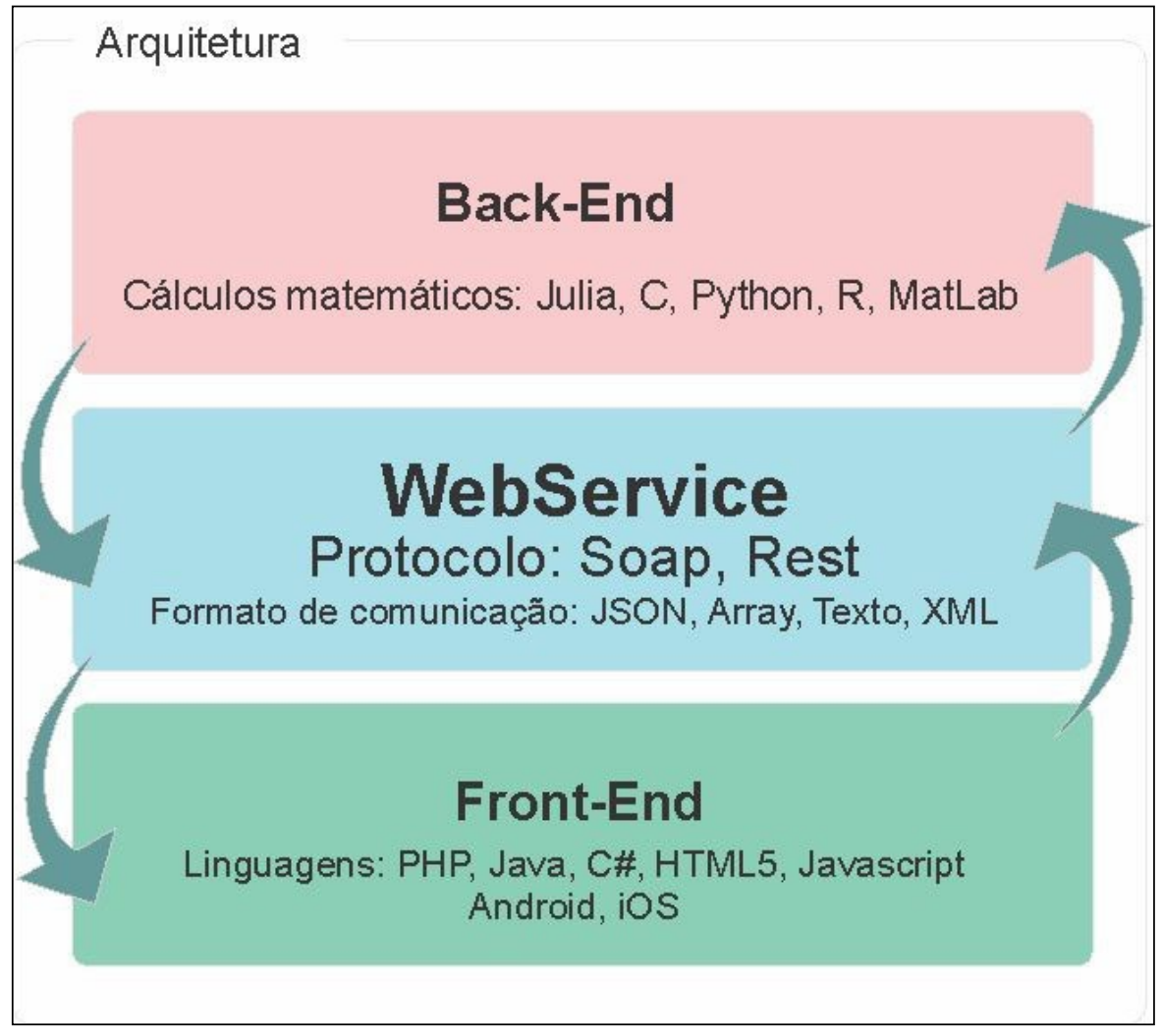

Fonte: Dos autores (2017).

A camada de back-end, responsável pelos cálculos, conforme a complexidade que configura o cenário, pode ser implementada sobre uma ou mais linguagens de programação. Como o lançamento de projétil oblíquo não requer grandes esforços computacionais, foi escolhida a linguagem $\mathrm{C} / \mathrm{C}++$ (FELICIANO, 2010), que conta com bibliotecas matemáticas que facilitam o cálculo de variáveis com ângulos.

Para a camada de front-end, foi utilizado o framework bootstrap ${ }^{8}$, conhecido no mercado pela facilidade na criação de telas. Também alguns recursos da linguagem de programação PHP (Hypertext Preprocessor) foram necessários.

Para a comunicação entre ofront-end e back-end, os webservices permitem a comunicação entre diferentes sistemas, os quais atuam como uma interface

8 É um framework front-end que facilita a vida dos desenvolvedores web a criar sites com tecnologia mobile (responsivo). 
de comunicação. O webService tem a função de integrar sistemas e auxiliar na comunicação entre aplicações diferentes. Permite que novas aplicações se comuniquem com outros sistemas legados já existem e que sistemas desenvolvidos em plataformas diferentes sejam compatíveis. Os webservices são componentes que permitem diferentes aplicações enviar e receber dados em diferentes formatos (MORO et al., 2011).

$\mathrm{O}$ webservices estabelece comunicação com o hardware através de comunicação REST (Representational StateTransfer), que é qualquer comunicação sobre HTTP (Protocolo de Transferência de Hipertexto) que não tenha nenhuma camada no meio. O REST favorece a compatibilidade, e pode ser utilizado em dispositivos com baixa capacidade de processamento e recursos, pois se trata de simples requisições HTTP (WEBSREST, 2011).

Figura 2: Visão macro da comunicação entre as diferentes camadas do simulador

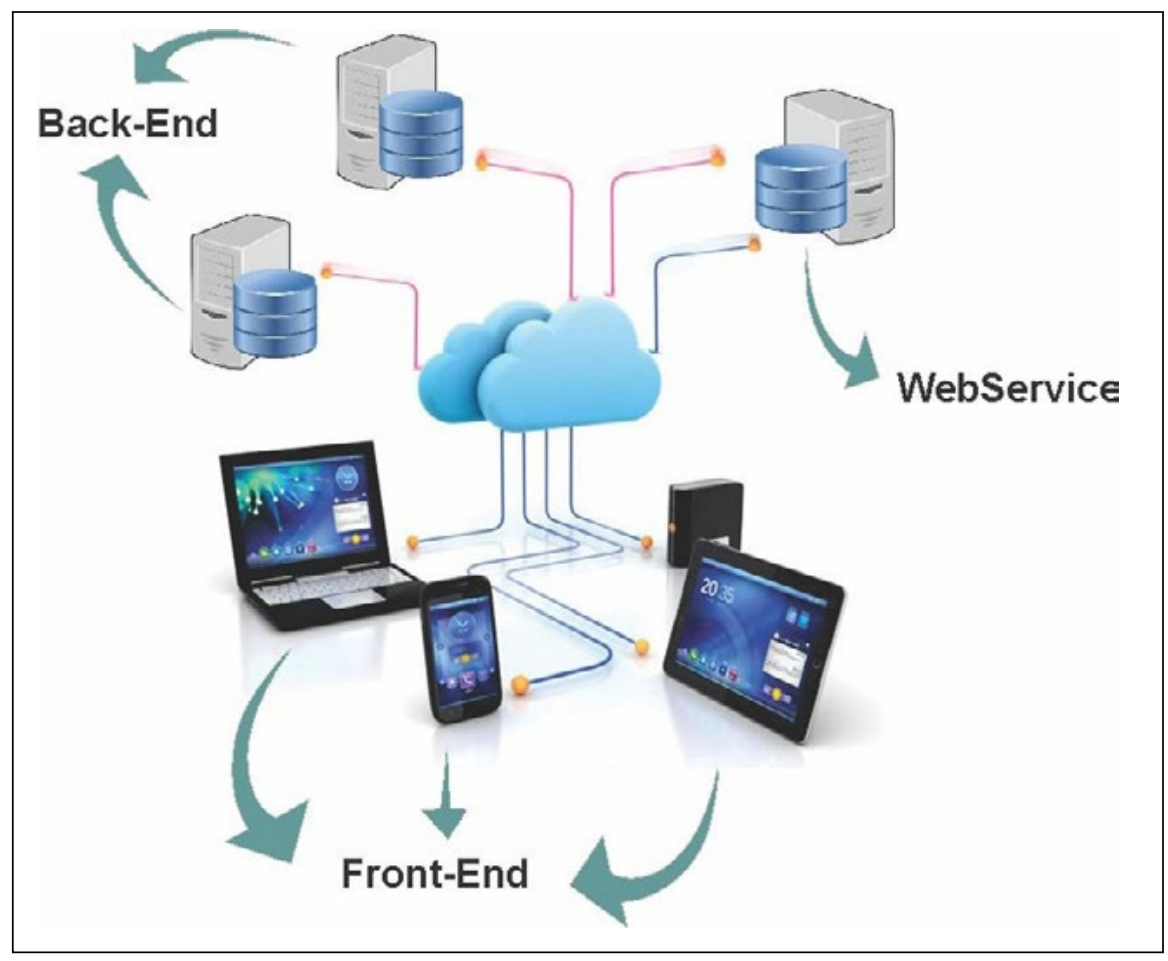

Fonte: Dos autores (2017).

Nesse contexto de interação entre front-end e back-end, o webservice irá cumprir a função de conectar as diferentes aplicações de simulação com o servidor remoto, desta forma diferentes implementações de simuladores podem utilizar o mesmo servidor para executar cálculos mais complexos. 


\subsection{Construção do simulador de lançamento oblíquo}

Provando que a arquitetura proposta é funcional, o simulador foi construído utilizando linguagens diferentes em cada camada e a camada de front-end e backend, ligadas por meio de um webservice.

Em especial, o back-end foi desenvolvido em C (Linguagem de programação), e envolve questões sobre o lançamento oblíquo de um projétil, no qual a resistência do ar é desprezível. São abordadas situações do comportamento de um lançamento de um objeto que é lançado para cima e para frente. Para a realização dos cálculos, é necessário informar três parâmetros de entrada (velocidade inicial, ângulo de saída e a gravidade do local).

Para os cálculos de altura máxima e tempo do objeto no ar, foi utilizado a biblioteca math.h, disponível no C, que fornece funções matemáticas básicas. São disponibilizadas, por exemplo, funções trigonométricas como o cosseno (cos), que opera com ângulos medidos em radianos, função para cálculo de raiz quadrada (sqrtf) entre outras. Essa biblioteca facilitou a programação dos cálculos como pode ser verificada na Figura 3.

Figura 3: Exemplo do código fonte envolvendo cálculo de física escrito na linguagem $C$, usando as funções da biblioteca math.h.

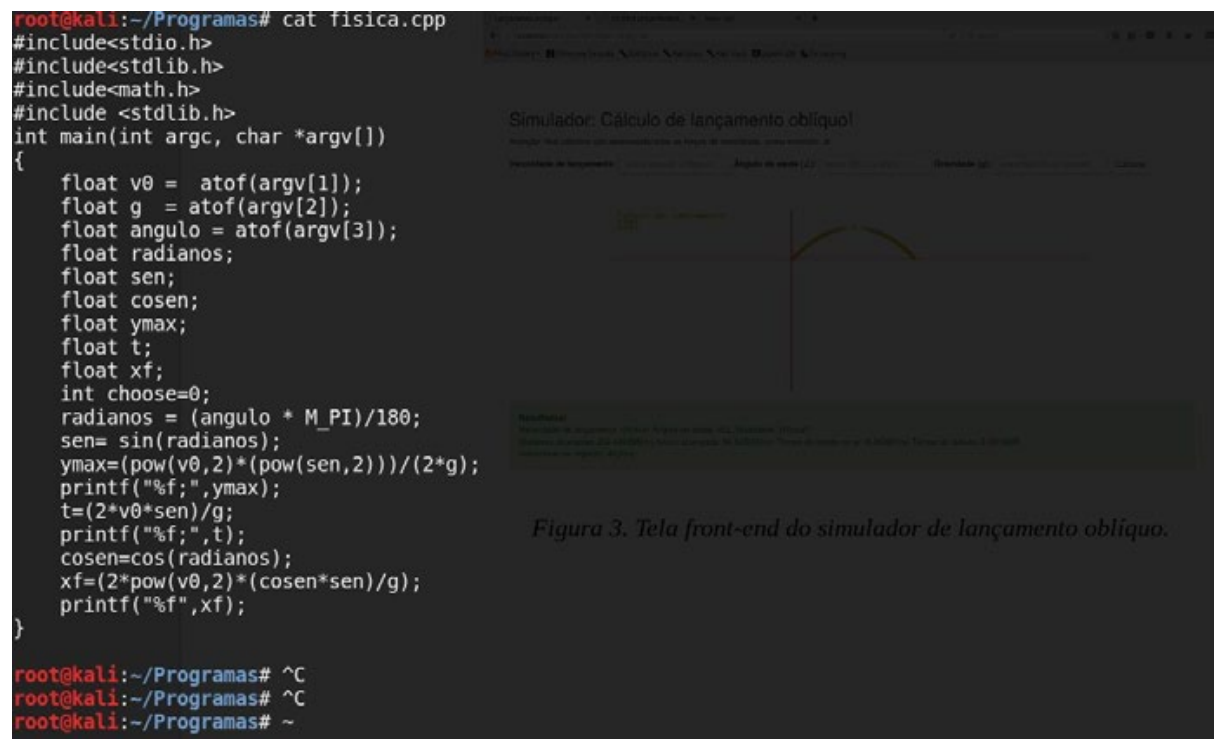

Fonte: Dos autores (2017).

Na Figura 3, conforme demonstrado no algoritmo de Pinheiro (2009) pode aplicar as funções para cálculos envolvendo trigonometria em C sempre em radianos. Como exemplos, apresentamos o trecho de código: 
A linha de programação: $y \max =(\operatorname{pow}(v 0,2) *(p o w(\operatorname{sen}, 2))) /\left(2^{*} g\right)$, é o mesmo que, alcance vertical $(\mathrm{m})=\mathrm{Vo}^{2 *} \operatorname{sen} \theta^{2} /\left(2^{*} \mathrm{~g}\right)$, (onde velocidade inicial $\mathrm{v}_{0}(\mathrm{~m})$ e aceleração da gravidade $\left.\mathrm{g}\left(\mathrm{m} / \mathrm{s}^{2}\right)\right)$.

Da mesma forma, a linha $\mathbf{x f}=\left(\mathbf{2}^{*}\right.$ pow $\left.(\mathbf{v} 0,2) *(\operatorname{cosen} * \mathbf{s e n}) / \mathbf{g}\right)$ corresponde ao alcance final horizontal $(\mathrm{m})=2^{*} \mathbf{V o}^{*}\left(\operatorname{sen} \theta^{*} \cos \theta\right) / \mathrm{g}$, onde velocidade inicial é $\mathrm{v} 0(\mathrm{~m})$ e aceleração da gravidade é $\mathrm{g}\left(\mathrm{m} / \mathrm{s}^{2}\right)$,

E por último a linha $\mathbf{t}=\left(\mathbf{2}^{*} \mathbf{v} \mathbf{0}^{*} \mathbf{s e n}\right) / \mathbf{g}$; é o mesmo que o tempo do objeto $\operatorname{not}(\mathbf{s})=\left(2^{*} \mathbf{v o}{ }^{*} \operatorname{sen} \theta\right) / g$, onde velocidade inicial $v 0(m)$ e aceleração da gravidade é $\mathrm{g}\left(\mathrm{m} / \mathrm{s}^{2}\right)$.

A camada de front-end, levando-se em consideração o conhecimento pré-existente, foi o mais fácil de ser construído. Resumiu-se em uma página da internet, composta por três caixas de texto, que solicita ao usuário o ângulo de saída, velocidade inicial e gravidade do local, e um botão para realizar a simulação. Essa camada não realiza nenhum tipo de cálculo, logo, as informações solicitadas ao usuário serão enviadas ao back-end.

Para o funcionamento do simulador, a construção do webservice, item mais importante e responsável pela integração das camadas descritas acima, foi desenvolvida em PHP linguagem flexível e amplamente utilizada no mercado. Para a camada de comunicação utilizou-se do protocolo SOAP (Simple Object Access Protocol), que facilita a integração entre componentes distintos (CURBERA, 2013).

\subsection{Fenômenos da Física no lançamento oblíquo}

Quando um objeto é lançado, de forma oblíqua sob um determinado ângulo entre o vetor velocidade e a direção $x$ igual a zero, podemos observar que ele realiza um movimento parabólico. Neste caso especial, o vetor velocidade inicial possui apenas a componente $\mathrm{v}_{\mathrm{x}^{\prime}}$, sendo a componente $\mathrm{v}_{\mathrm{y}}$ nula. Já quando o corpo é lançado a partir do solo em um determinado ângulo com a horizontal, que não seja zero, o movimento é classificado como lançamento oblíquo, com o vetor velocidade inicial possuindo ambas as componentes, $\mathrm{v}_{\mathrm{x}} \mathrm{e}$ $\mathrm{v}_{\mathrm{y}^{\prime}}$ diferentes de zero. Em ambos os casos, a análise dos movimentos despreza as forças dissipativas, sendo que o corpo fica sujeito apenas à ação da gravidade, descrevendo, portanto, uma trajetória parabólica.

Conforme a Figura 4, existe dois movimentos envolvidos no lançamento oblíquo, o movimento na direção do eixo $x$, que é uniforme, pois a velocidade é constante e o movimento na direção do eixo y, no qual a velocidade aumenta pela ação do campo gravitacional terrestre. 
Figura 4: Comportamento do movimento do lançamento oblíquo em x e y.

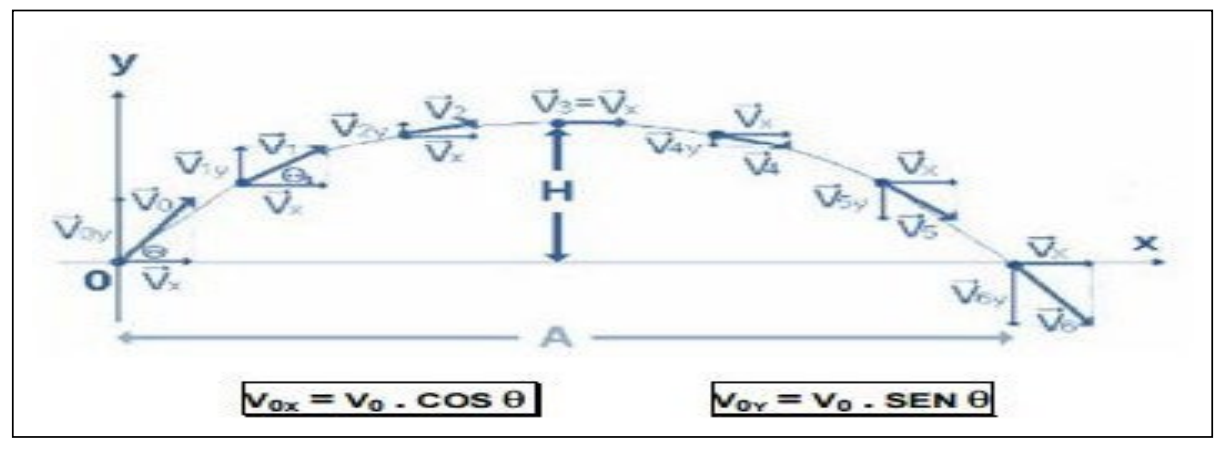

Fonte: Dos autores (2017).

Conforme Sousa (SOUSA, 2013), o movimento desse objeto é o resultado de dois movimentos: o movimento vertical uniformemente variado (que é um movimento de lançamento vertical e em seguida um movimento de queda livre) cuja aceleração é a da gravidade, e o movimento horizontal uniforme, sendo que na horizontal não há aceleração e o corpo se movimenta por inércia. Esses movimentos são simultâneos e independentes.

Com tudo isso, percebemos que para calcular o lançamento oblíquo, precisamos identificar a velocidade inicial, o valor da gravidade local, o tempo entre o lançamento e a chegada ao destino e a distância percorrida ou alcance do projétil. A partir destas informações, fica mais fácil a identificação de quais serão as equações utilizadas para o cálculo.

Após finalizar a construção do simulador, este foi disponibilizado para um grupo de pessoas com conhecimento na área de física para testar a usabilidade. Em todos os casos os usuários conseguiram utilizar o simulador, inclusive em dispositivos móveis. Alguns usuários questionaram sobre a possibilidade de simular outros cálculos de Física, o que é um bom indicador de que o simulador foi aprovado. 
Figura 5: Tela front-end do simulador de lançamento oblíquo.

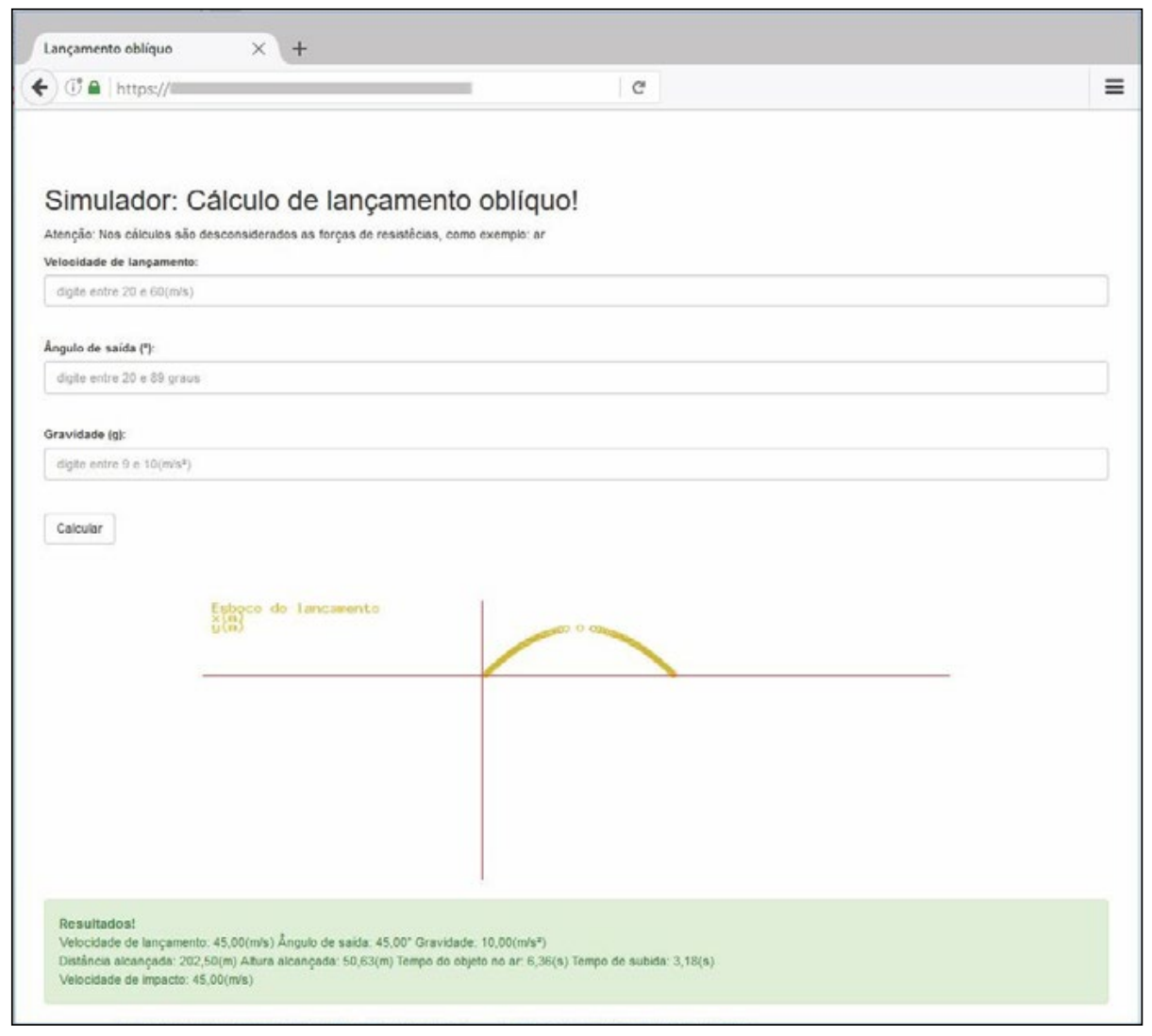

Fonte: Dos autores (2017).

A Figura 5 apresenta a tela que o usuário utiliza para entrada de dados e visualização das respostas da simulação para os parâmetros de alcance, tempo de permanência no ar e altura máxima, entre outros. O simulador é acessível pelo navegador no link (https:/ /infocos.com.br/fisica/ fisica.php).

\subsection{Estrutura do objeto de estudo de hidrostática}

Para o desenvolvimento do Objeto de Aprendizagem envolvendo hidrostática foi utilizada a plataforma de desenvolvimento Scratch, que é uma linguagem gráfica de programação criada no Media Lab do Instituto de Tecnologia de Massachusetts, inspirada nas linguagens Logo e Squeak, mas que pretende ser mais simples, fácil de utilizar e mais intuitiva (SCRATCH, 2012). $\mathrm{O}$ objeto foi desenvolvido e validado, e necessitaram de um conhecimento considerável para a utilização da ferramenta Scratch, e um conhecimento básico na área de estática dos fluidos. Por meio de uma solução web, ou seja, 
acessível via navegador de Internet, é possível simular com alguns parâmetros customizáveis o comportamento dos objetos jogados no líquido.

\subsection{Construção do objeto de estudo}

Para demonstrar que é possível criar objetos de aprendizagem com Scratch, inicialmente será explicado de maneira sucinta como foi o desenvolvimento deste objeto.

Na Figura 6 é apresentada a programação do Scratch. No lado direito, a mesma é feita através de caixas que são encaixados uns aos outros, formando uma sequência de comandos. Os blocos são concebidos para se encaixar apenas de uma única forma, fazendo sentido sintaticamente, não ocorrendo, assim, erros de sintaxe. É uma maneira de programar um objeto em alto nível para seus usuários.

O Scratch permitiu que fosse criado o programa estilo passo-a-passo, primeiramente inicia-se o programa com a declaração de todas variáveis, seguindo com a entrada de dados pelo usuário. Com os dados de entrada já em variáveis, é possível realizar cálculos para saber se os blocos vão afundar ou não.

Figura 6: Programação interativa de alto nível do Scratch.

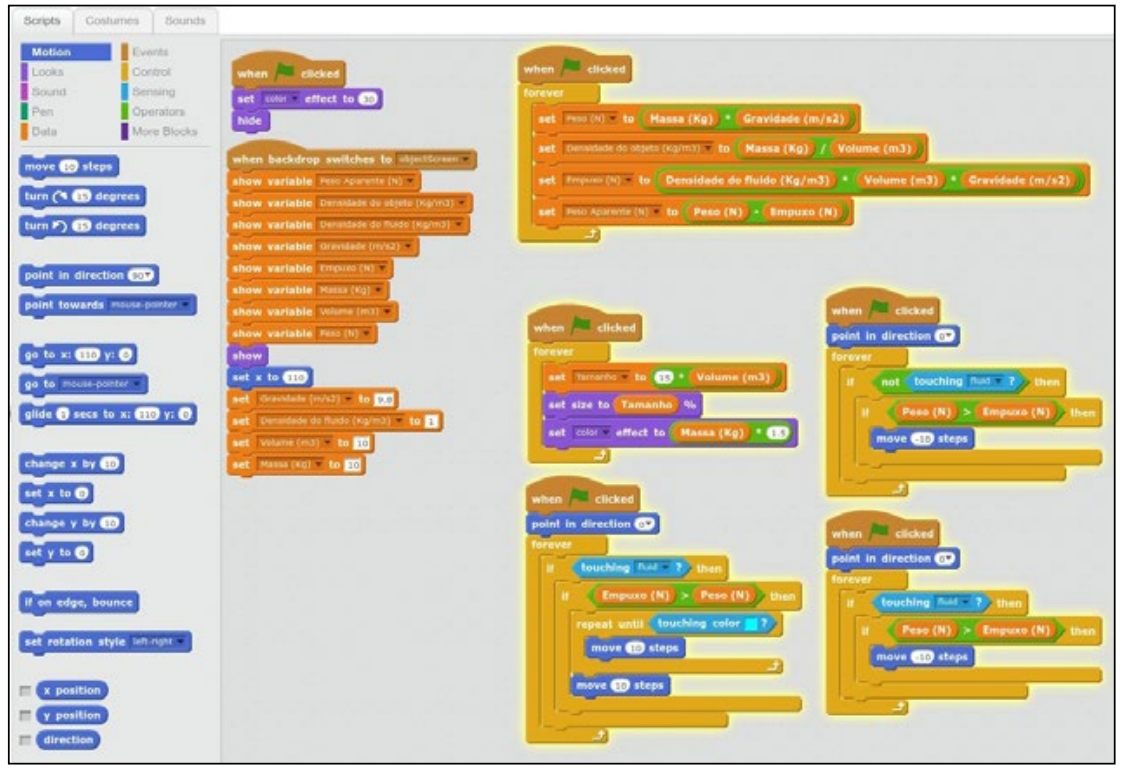

Fonte: Dos autores (2017). 


\subsection{A Física na estática dos fluidos}

Um sábio grego Arquimedes (282-212 a.C) enquanto tomava banho, descobriu que um corpo imerso na água se torna mais leve devido a uma força exercida pelo líquido sobre o corpo, vertical e para cima, que alivia o peso do corpo. Essa força, do líquido sobre o corpo, é denominada empuxo, e tem origem na pressão diferencial exercida pelo fluido na parte superior e inferior do objeto.

Portanto, em um corpo que se encontra imerso em um líquido, agem duas forças: a força peso, devida à interação com o campo gravitacional terrestre, e a força de empuxo, devida à sua interação com o líquido.

Quando um corpo está totalmente imerso em um líquido, podemos ter as seguintes condições:

- Se a intensidade da força de empuxo é igual à intensidade da força peso $(E=P)$, ele permanece parado no ponto onde foi colocado;

- Se a intensidade da força de empuxo é menor do que a intensidade da força peso $(\mathrm{E}<\mathrm{P})$, ele afunda;

- Se a intensidade da força de empuxo é maior do que a intensidade da força peso $(\mathrm{E}>\mathrm{P})$, ele é levado para a superfície, ali atingindo o equilíbrio, uma vez que o volume de líquido deslocado passa a ser menor que o volume total do corpo e, portanto, peso e empuxo passam a se equilibrar.

Em física, o empuxo é uma força de reação descrita quantitativamente pelas segunda e terceira leis de Newton (CORRADI, 2010). Quando um sistema expele ou acelera massa em uma direção, a massa acelerada vai causar uma força de igual magnitude, mas em sentido oposto.

Foi o filósofo, matemático, físico, engenheiro, inventor e astrônomo grego Arquimedes quem descobriu como calcular o empuxo. $\mathrm{O}$ Empuxo pode ser calculado pela equação, que corresponde à concepção de que o empuxo é igual ao peso de fluido deslocado:

$$
\begin{aligned}
& \mathbf{E}=\rho^{*} \mathbf{V}^{*} \mathbf{g} \\
& \text { Onde: } \\
& \mathrm{E}=\text { Empuxo }\left(\mathrm{N}=\mathrm{kg} \cdot \mathrm{m} / \mathrm{s}^{2}\right) \\
& \rho=\text { Densidade }\left(\mathbf{k g} / \mathrm{m}^{3}\right) \mathbf{V}=\operatorname{Volume}\left(\mathbf{m}^{3}\right) \\
& \mathrm{g}=\text { Aceleração da Gravidade }\left(\mathrm{m} / \mathrm{s}^{2}\right)
\end{aligned}
$$

\subsection{Objeto de aprendizagem: hidrostática}

Tendo apresentado um modelo de utilização da ferramenta criada, pode-se perceber que o Scratch é uma ferramenta que pode auxiliar os alunos da disciplina de Linguagem de Programação e Algoritmos que possuem 
dificuldade em organizar pensamentos, em construir conceitos matemáticos, ou que evidenciam problemas com informática.

Como afirma Pereira et al. (2012), essa plataforma pode ser utilizada pelos estudantes no início da disciplina, com isso terão melhor compreensão dos conceitos ensinados em aula. Tal aspecto permite exercitar a criatividade e o raciocínio científico, lógico e matemático, tornando o aluno apto a solucionar qualquer problema computacional que lhe seja apresentado, assim como comprovado na criação do objeto de aprendizagem de hidrostática.

Conforme visto na Figura 7, são exigidas interações do usuário, informando parâmetros como a massa $(\mathrm{kg})$, o volume da caixa $\left(\mathrm{m}^{3}\right)$, entre outros. Com essas informações, o simulador tem condições de determinar se a caixa irá ou não afundar no líquido pré-definido.

O usuário ainda pode simular várias ações em tempo real, observando o comportamento do bloco em relação à densidade da água.

Figura 7: Tela do objeto de aprendizagem sobre hidrostática.

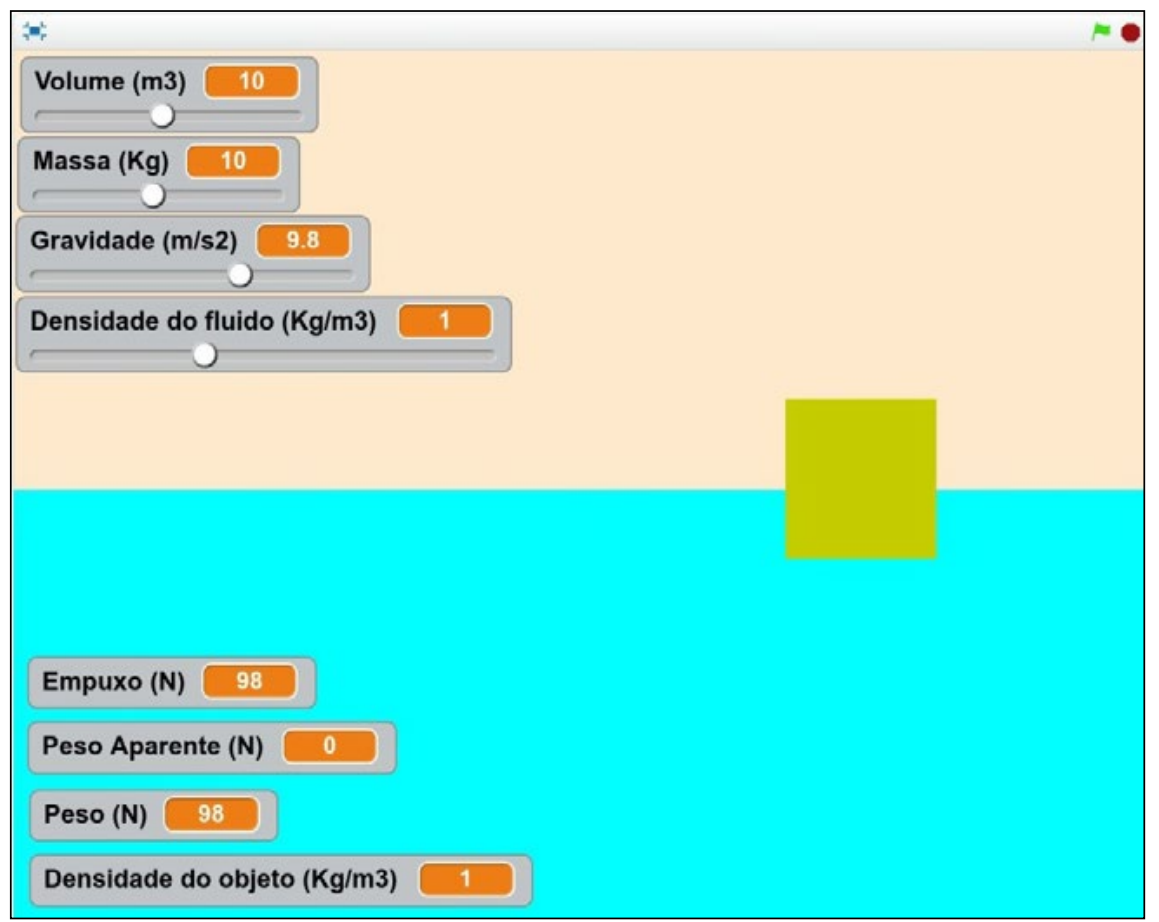

Fonte: Dos autores (2017).

A ferramenta roda no navegador, o que permite que o objeto criado possa ser disponibilizado para outros usuários estudarem a forma como foi construída a ferramenta, testar e simular a entrada de diversos parâmetros. A ferramenta pode ser acessada pelo link (https://scratch.mit.edu/projects/132597221/). 


\section{O OLHAR DOS ESTUDANTES ENVOLVIDOS NAS ATIVIDADES}

Por meio dos objetos desenvolvidos os estudantes relatam que perceberam os benefícios do ensino baseado na construção de objetos, a interdisciplinaridade que esse método promoveu, pois tiveram que aliar conhecimentos de física, de cálculo e de programação. Também destacam a aprendizagem significativa de saberes, que também se tornaram mais críticos em relação às suas decisões, e ainda, apontam o desenvolvimento de competências e habilidades para resolução de problemas e trabalho em equipe.

Os benefícios da atividade já se evidenciaram antes mesmo da implementação, pois exigiu do grupo contextualizar problemas e procurar por soluções, fazendo com que os integrantes se posicionassem de forma crítica e argumentativa diante de cada decisão, tendo sempre em vista as estratégias e ferramentas disponíveis para cada solução, conforme os objetivos a serem alcançados.

Muito além do conhecimento de Física que foi proposto em sala de aula a ser aplicado, exigiu um conhecimento profundo de várias outras áreas, tais como a lógica, a Matemática, as linguagens de programação, conhecimentos gerais de TI, modelagem de dados, IHC (Interação-Humano Computador), paradigmas de programação entre outros, evidenciando a necessidade de uma abordagem multidisciplinar para a realização da atividade

Pensando em disponibilizar os Objetos de Aprendizagem confeccionados para auxiliar na construção do conhecimento para outras pessoas, surgiram outras preocupações, tais como a usabilidade, a disponibilidade/acessibilidade, a confiabilidade e precisão dos resultados, promovendo novos problemas a serem solucionados. Diante desse quadro de desafios percebeu-se que é mais fácil o trabalho em grupo para resolução de problemas.

Dentre as dificuldades que foram superadas, destaca-se a do entendimento ao raciocínio lógico, melhoria significativa e sólida base de conceitos matemáticos e lógico-matemático e principalmente da Física envolvida na confecção dos objetos de aprendizagem.

\section{CONSIDERAÇÕES FINAIS}

Por meio da atividade proposta foi possível perceber que, confeccionar objetos de estudos digitais aumenta significativamente a percepção cognitiva e a formação dos conceitos de Física e lógica nos estudantes. Verificou-se também a boa aceitação pelos estudantes e a motivação, observada nas aulas durante a confecção dos objetos de aprendizagem.

Quanto ao aspecto da aprendizagem destaca-se que as simulações exigiram dos estudantes a revisitação a diversos conceitos e equações da Física e, além disso, adaptá-los a linguagem da programação. Este foi um dos aspectos que os motivou para seguir e compreender os fenômenos que ali 
estavam envolvidos. Isso pode ser comprovado em alguns objetos, no qual os estudantes tiveram que buscar conceitos e equações que não estavam previstas na disciplina, mas que o objeto desenvolvido exigiu para um bom êxito da simulação.

O desenvolvimento desta atividade também possibilitou estabelecer pontes entre conceitos de diferentes áreas do conhecimento, estimulando relações e conexões interdisciplinares que potencializam a formação e a aprendizagem dos estudantes. Neste sentido, Ketzer (2007) aponta que a ênfase do ensino tradicional, a decoreba sem aplicação está desarticulada da real compreensão para a utilização dos conceitos em situações práticas da vida. Problemas que emergem na sociedade, em nosso dia a dia requerem um olhar diferenciado, com conhecimentos de diferentes áreas do saber, portanto experiências como esta que desafiam os estudantes a pensar sobre um problema real os aproxima da realidade, os tornam mais críticos e autônomos na busca pelas soluções, além de aproximá-los mais de sua futura profissão.

\section{REFERÊNCIAS}

BRANDÃO, R. V.; ARAUJO, I. S.; VEIT, E. A. A modelagem científica de fenômenos físicos e o ensino de física. A Física na Escola, São Paulo, v. 9, n. 1, p. 10-14, maio. 2008

CERT.BR, 2015 Cert.br, Cartilha de segurança para Internet. Disponível em: <http:/ / cartilha.cert.br/criptografia/> Acessado em out. 2016.

CORRADI, V. Fundamentos de física - Editora UFMG, 2010.

CURBERA, 2013 DUFTLER Matthew, KHALAF Rania. Unraveling the Web services web: an introduction to SOAP, WSDL, and UDDI. Disponível em: <http:/ /www. immagic.com/eLibrary/ARCHIVES/SUPRSDED/W3C/W000520N.pdf> Acessado em out. 2016.

FELICIANO, G. 2010 Linguagem de programação C/C++. Disponível em: <http:/ / www.ceavi.udesc.br/arquivos/id_submenu/387/patricia_felicianoguilherme_cesar_ lamego.pdf> Acessado em out. 2016.

KETZER, Solange Medina. Ensinar e aprender no jogo da interdisciplinaridade. In: AUDI, Jorge Luis Nicolas; MOROSINI, Marilia Costa. Inovação e interdisciplinaridade na Universidade. Porto Alegre: EDIPUCRS, 2007, p. 91-100.

MOREIRA, M. A. Modelos científicos, modelos mentais, modelagem computacional e modelagem matemática: aspectos epistemológicos e implicações para o ensino.

Revista Brasileira de Ensino de Ciência e Tecnologia, v. 7, n. 2, p. 1-20, 2014.

PEREIRA, P. S.; MEDEIROS, M. MENEZES, J. W. M. Análise do Scratch como ferramenta de auxílio ao ensino de programação de computadores. Anais do XL Congresso Brasileiro de Educação em Engenharia. 2012. Disponível em: <http:/ / 
www.abenge.org.br/CobengeAnteriores/2012/artigos/104281.pdf > Acessado em out. 2016.

SCRATCH, 2012. About Scratch (Scratch Documentation Site). Disponível em: <http://info.scratch.mit.edu/About_Scratch> Acessado em out. 2016.

SILVA, N.G.; COTTA, J. Aplicação do cálculo no lançamento de projéteis. EVINCI Evento de Iniciação Científica. v.1, nº 3, 2015. Disponível em: http:/ / portaldeperiodicos.unibrasil.com.br/index.php/anaisevinci/article/view/807. Acessado em out. 2016.

SOUSA, H. de S. Alguns aspectos acerca do conceito de lançamento oblíquo, em análise a parábola de segurança. Monografia, Universidade Estadual do Ceará, 2013. Disponível em: <http:/ / www.uece.br/fisica/index.php/arquivos/doc_ download/172->Acessado em out. 2016.

VEIT, E. A.; TEODORO, V. D. Modelagem no ensino/aprendizagem de física e os novos parâmetros curriculares nacionais para o ensino médio. Revista Brasileira de Ensino de Física, 2002. v. 24, n. 2, p. 87-96.

MORO, T.D; DORNELES, C.F.; REBONATTO, M.T. Webservices WS - versus Web Services REST. REIC - Revista de Iniciação Científica, v 11, $\mathrm{n}^{\circ}$ 1, 2011. Disponível em: <http:/ / www.seer.ufrgs.br/reic/article/viewFile/22140/12928> Acessado em out. 2016.

WILEY, D.A. (2000). “Connecting learning objects to instructional design theory: A definition, a metaphor, and a taxonomy". In: WILEY, D.A. (Org.). The Instructional Use of Learning Objects. Disponível em: <http:/ / reusability.org/read/chapters / wiley.doc>. Acesso em: 7 jun. 2004. 